\title{
Effects of the Calcium-Activated Chloride Channel Inhibitors T16Ainh-A01 and CaCCinh-A01 on Cardiac Fibroblast Function
}

\author{
Xiang-qin Tian ${ }^{\mathrm{a}, \mathrm{b}} \quad$ Ke-tao Ma $\mathrm{Ma}^{\mathrm{a}} \quad$ Xian-wei Wang ${ }^{\mathrm{b}} \quad$ Yang Wang $^{\mathrm{a}} \quad$ Zhi-kun Guo $^{\mathrm{b}}$ \\ Jun-qiang $\mathrm{Si}^{\mathrm{a}}$ \\ aDepartment of Physiology, Medical College of Shihezi University, Shihezi, 'Henan Key Laboratory of \\ Medical Tissue Regeneration, Xinxiang Medical University, Xinxiang, China
}

\section{Key Words}

Anoctamin-1 - Calcium-activated chloride channels $•$ Cardiac fibroblasts $•$ Cardiac fibrosis

\begin{abstract}
Background/Aims: Calcium-activated chloride channels (CaCCs) regulate numerous physiological processes including cell proliferation, migration, and extracellular matrix secretion. T16Ainh-A01 and CaCCinh-A01 are selective inhibitors of CaCCs. But it is unknown whether these two compounds have functional effects on cardiac fibroblasts (CFs). Methods: Primary CFs were obtained by enzymatic dissociation of cardiomyocytes from neonatal rat hearts. Intracellular $\mathrm{Ca}^{2+}\left(\left[\mathrm{Ca}^{2+}\right]_{\mathrm{i}}\right)$ and $\mathrm{Cl}^{-}\left(\left[\mathrm{Cl}^{-}\right]_{i}\right)$ were measured using the fluorescent calcium indicators (Fluo-4 $\mathrm{AM}$ ) and $\mathrm{N}$-(ethoxycarbonylmethyl)-6-methoxyquinolinium bromide respectively. The expression of anoctamin-1 (ANO1) and $\alpha$-smooth muscle actin ( $\alpha$-SMA) was detected by quantitative RT-PCR, immunofluorescence, and western blotting. A hydroxyproline assay was used to examine collagen secretion. Cell proliferation, cell cycle distribution, and cell migration were assessed by Cell Counting Kit-8, flow cytometry, and Transwell assays, respectively. Results: ANO1 was preferentially expressed on the nuclear membrane and partially within intracellular compartments around the nucleus. T16Ainh-A01 and CaCCinh-A01 displayed different inhibitory effects on $\left.\mathrm{CCl}^{-}\right]_{i}$ in CFs. T16Ainh-A01 considerably decreased $\left[\mathrm{Cl}^{-}\right]_{i}$ in the nucleus, whereas CaCCinh-A01 reduced $\left[\mathrm{Cl}^{-}\right]_{i}$ in intracellular compartments around the nucleus, and both inhibitors exhibited a minimal effect on $\left[\mathrm{Ca}^{2+}\right]_{\mathrm{i}}$ in CFs. ANO1 and $\alpha$-SMA expression levels were significantly repressed by CaCCinh-A01. T16Ainh-A01 showed a marked inhibitory effect on the mRNA levels of ANO1 and $\alpha$-SMA, but had a negligible effect on ANO1 at the protein level. T16Ainh-A01 and CaCCinh-A01 led to the significant repression of cell proliferation, cell migration, and collagen secretion in CFs. Conclusion: Our findings
\end{abstract}

Dr. Jun-qiang Si

and Dr. Zhi-kun Guo
Department of Physiology, Medical College of Shihezi University

106 North St, Shihezi 832002 (China)

E-Mail sijunqiang11@hotmail.com; gzk@xxmu.edu.cn 


\section{Cellular Physiology Cell Physiol Biochem 2018:49:706-716

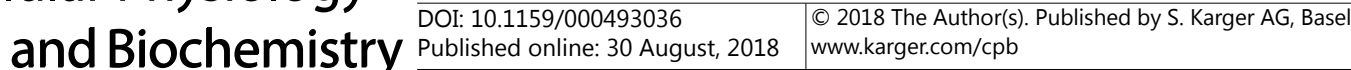 \\ Tian et al.: Effects of Cacc Inhibitors on CF Function}

indicate that T16Ainh-A01 and $\mathrm{CaCCinh}-\mathrm{A} 01$ have the potential to inhibit the proliferation and collagen secretion of CFs and may serve as novel anti-fibrotic therapeutic drugs in the future.

(C) 2018 The Author(s)

Published by S. Karger AG, Basel

\section{Introduction}

Cardiac fibroblasts (CFs) are located in interstitial and perivascular matrices and are essential for homeostasis [1,2]. CFs are not only involved in extracellular matrix protein production, tissue repair, scar formation, inflammatory response, vasculogenesis, and tumorigenesis, but also have a role in myocardial remodeling, hypertensive heart disease, and heart failure $[3,4]$. CFs actively participate in the regulation of cardiac physiological and pathological processes and thus represent pertinent targets for pharmacological approaches for cardiac diseases, which may point to novel therapeutic strategies [5].

Ion channels participate in diverse types of physiological activity integral to excitability, contraction, cell cycle, salt and water balance, and metastatic cascades, which play significant roles in the maintenance of tissue homeostasis during cell proliferation, differentiation, and apoptosis [6]. Normal cardiac electrophysiology relies on the dynamic and orchestrated operation of membrane ion channels and intracellular ion regulatory machineries [7]. The fibroblast properties of migration, secretion, and proliferation can be regulated by the activation of ion channels [5]. Meanwhile, chloride channels play essential roles in a variety of physiological functions including epithelial secretion, smooth muscle contraction, and sensory transduction [8]. Increasing evidence indicates that calcium-activated chloride channels (CaCCs) may participate in CF function [9-11] and be potential drug targets for the treatment of cardiovascular diseases [12].

Anoctamin-1 (ANO1; also known as TMEM16A, DOG1, ORAOV2, and TAOS2) is an eighttransmembrane domain protein functioning as a CaCC in the plasma membrane [13-15]. T16Ainh-A01 and CaCCinh-A01 are inhibitors of CaCCs that exhibit differences in their specific suppression of distinct cells. These compounds display poor selectivity for ANO1 and inhibition of CaCCs in vascular tissue in the concentration range that inhibits isolated conductance [16]. CaCCinh-A01 can inhibit AN01-dependent chloride conductance and decrease the proliferation of ANO1-dependent cancer cell lines (Te11 and FaDu cells) by degrading AN01 protein, whereas T16Ainh-A01 has no effect on ANO1 protein, although it exhibits anti-proliferative effects on cell lines (human airway and intestinal cells) with extremely low ANO1 expression levels $[17,18]$. Furthermore, CaCCinh-A01 reduces cell viability, whereas T16Ainh-A01 has a minimal effect on the viability of colon cancer cells [19]. The inhibitory effects of T16Ainh-A01 and CaCCinh-A01 on CaCCs might be mediated by either the same or distinct signaling pathways in a cell type-specific manner. However, it is unclear whether both compounds influence the function of CFs. The aim of this study was to ascertain the effects of T16Ainh-A01 and CaCCinh-A01 on ANO1 expression, distribution of $\mathrm{Ca}^{2+}$ and $\mathrm{Cl}^{-}$, cell proliferation, cell migration, and collagen secretion in CFs.

\section{Materials and Methods}

\section{Animals}

Neonatal Sprague-Dawley rats (male, 1-2 days old) were obtained from the Center of Experimental Animals of Xinxiang Medical University. Animal treatment was performed according to the guidelines of the Ministry of Science and Technology of the People's Republic of China ([2006]398) and approved by the Xinxiang Medical University Animal Care Committee (No. 030032).

Drugs and reagents

T16Ainh-A01(2-[(5-ethuy1-1,6-dihydro-4-methy1-6-oxo-2-pyrimidiny1)thio]-N-[4-4(4methoxypheny1)-2-thiazoyoly1]acetamide $)$ and CaCCinh-A01 (6- $(1$, 1-dimethylethy1)-2-[(2furanylcarbonyl)amino]-4, 5,6, 7-tetrahydrobenzo [b]thiophene-3-carboxylic acid) were purchased from 


\section{Cellular Physiology Cell Physiol Biochem 2018:49:706-716

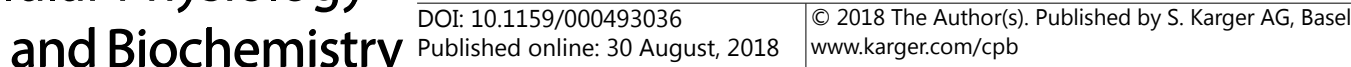

Tian et al.: Effects of Cacc Inhibitors on CF Function

Sigma-Aldrich (St. Louis, MO, USA). Stock solutions of the above reagents (20 mM) were prepared using dimethyl sulfoxide (DMSO) as a solvent, and stored at $-20^{\circ} \mathrm{C}$ until use. All work solutions were prepared freshly from stock solutions before each experiment and not exposed to light. CFs were treated with $10 \mu \mathrm{M}$ T16Ainh-A01, $30 \mu \mathrm{M}$ CaCCinh-A01, and matching volumes of DMSO $(0.1 \% \mathrm{v} / \mathrm{v})$.

\section{CF isolation and culture}

Primary CFs were obtained from the left ventricles of neonatal Sprague-Dawley rats by enzymatic dissociation and selective plating methods as described previously [20, 21]. In brief, neonatal rats were euthanized by $\mathrm{CO}_{2}$ asphyxiation. Their hearts were excised under sterile conditions and cut into approximately $1-\mathrm{mm}^{3}$ pieces. The tissues were rinsed in Hanks' balanced salt solution prior to digestion with $0.1 \%\left(\mathrm{w} / \mathrm{v}\right.$ ) collagenase type II (Sigma-Aldrich) in Hanks' balanced salt solution without $\mathrm{Ca}^{2+}$ and $\mathrm{Mg}^{2+}$ for $20 \mathrm{~min}$ and then $0.125 \%(\mathrm{w} / \mathrm{v})$ trypsin (Sigma-Aldrich) for $10 \mathrm{~min}$ at $37^{\circ} \mathrm{C}$ in a shaking bath. After the addition of Dulbecco's modified Eagle's medium (DMEM: ThermoFisher Scientific, Shanghai, China), the samples were filtered with a 70- $\mu \mathrm{m}$ metal mesh filter and centrifuged for $10 \mathrm{~min}$ at $1000 \times \mathrm{g}$. The filtered particles were resuspended in DMEM supplemented with $10 \%(\mathrm{v} / \mathrm{v})$ fetal bovine serum (Thermo Fisher Scientific) and 1\% penicillin/streptomycin (Solarbio, Beijing, China). Finally, the CFs were separated from myocytes and other cells by selective plating (adherence after $45 \mathrm{~min}$ in culture). The identity and purity of CFs were assessed by characteristic morphology and immunofluorescence staining for vimentin. CFs from the third passage were used in the subsequent experiments.

\section{Measurements of intracellular $\mathrm{Ca}^{2+}$ and $\mathrm{Cl}^{-}$}

The effects of T16Ainh-A01 and CaCCinh-A01 on intracellular $\mathrm{Ca}^{2+}\left(\left[\mathrm{Ca}^{2+}\right]_{\mathrm{i}}\right)$ were monitored by imaging Fluo-4 AM fluorescence according to the manufacturer's instructions. Briefly, Fluo-4 AM was solubilized in anhydrous DMSO and then diluted with HEPES-buffered saline (HBS). CFs with $60 \%$ confluence were washed 3 times with HBS and incubated with $10 \mu \mathrm{M}$ T16Ainh-A01, $30 \mu \mathrm{M}$ CaCCinh-A01, or 0.1\% DMSO for $48 \mathrm{~h}$. Following washing 3 times with HBS, the cells were incubated with Fluo-4 AM (2.5 $\mu \mathrm{M}, \mathrm{pH}$ 7.4) for $45 \mathrm{~min}$ at $37^{\circ} \mathrm{C}$ and washed 3 times with HBS. De-esterification of Fluo-4 AM was extended for an additional $20 \mathrm{~min}$. The excitation and emission wavelengths of Fluo-4 AM were 488 and $520 \mathrm{~nm}$, respectively. Background fluorescence measured prior to loading was subtracted from the measured emissions. Images were recorded with a laser scanning confocal microscope (LSM 510; Zeiss, Oberkochen, Germany).

The effects of T16Ainh-A01 and CaCCinh-A01 on intracellular $\mathrm{Cl}^{-}\left(\left[\mathrm{Cl}^{-}\right]_{\mathrm{i}}\right)$ were measured with the chloride probe N-(ethoxycarbonylmethyl)-6-methoxyquinolinium bromide (MQAE; Beyotime, Shanghai, China) as per the manufacturer's instructions. In brief, CFs at $60 \%$ confluence were washed 3 times with Krebs-HEPES buffer and incubated with $10 \mu \mathrm{M}$ T16Ainh-A01, $30 \mu \mathrm{M}$ CaCCinh-A01, or 0.1\% DMSO for $48 \mathrm{~h}$. Then, cells were incubated with $10 \mathrm{mM}$ MQAE for $1 \mathrm{~h}$ at $37^{\circ} \mathrm{C}$ in HEPES buffer before they were transferred to a perfusion chamber. The excitation and emission wavelengths of Fluo-4 AM were 355 and $460 \mathrm{~nm}$, respectively. Images were recorded with a laser scanning confocal microscope (LSM 510; Zeiss).

\section{RNA extraction and quantitative real-time RT-PCR ( $q R T-P C R$ )}

Primary CFs were isolated as described above, and approximately $1.0 \times 10^{6}$ cells were collected with a cell sorter. Total RNA was extracted from CFs with the TRIzol reagent (ThermoFisher Scientific, Shanghai, China) and quantified by assessing their optical densities at 260 and $280 \mathrm{~nm}$ using a NanoDrop ND-100 (LabTech, Beijing, China). Reverse transcription was performed according to the manufacturer's protocol (Thermo Fisher Scientific, Shanghai, China). In brief, $1 \mu \mathrm{L}$ total RNA (1-2 $\mu \mathrm{g}$ ) was added to reaction mixture containing $5 \times$ reaction buffer, $10 \mathrm{mM}$ dNTP mix, $1 \mu \mathrm{L}$ oligo(dT) ${ }_{18}$ primer, $20 \mathrm{U}$ RNase inhibitor, and $200 \mathrm{U}$ $\mathrm{M}-\mathrm{MuLV}$ reverse transcriptase to a final volume of $20 \mu \mathrm{L} \mathrm{cDNA}$ was synthesized at $42^{\circ} \mathrm{C}$ for $1 \mathrm{~h}$. Remaining enzymes were heat-inactivated at $70^{\circ} \mathrm{C}$ for $5 \mathrm{~min}$.

qRT-PCR was carried out with SYBR Premix Ex Taq ${ }^{\mathrm{TM}}$ II SYBR Premix Ex (TAKARA Bio, Inc., Dalian, China). All reactions during the process were amplified in triplicate on a LightCycler ${ }^{\circledR}$ System (Roche, Switzerland) in a total volume of $10 \mu \mathrm{L}$ containing $5 \mu \mathrm{L}$ of SYBR green master mix, $0.8 \mu \mathrm{L}$ of primer, $1 \mu \mathrm{L}$ diluted cDNA, and $3.2 \mu \mathrm{L} \mathrm{H}_{2} \mathrm{O}$. The samples were pre-incubated at $95^{\circ} \mathrm{C}$ for $10 \mathrm{~min}$, followed by 45 cycles of $95^{\circ} \mathrm{C}$ for $15 \mathrm{~s}, 61^{\circ} \mathrm{C}$ for $15 \mathrm{~s}$, and $72^{\circ} \mathrm{C}$ for $30 \mathrm{~s}$. The ratio between the amount of target genes and endogenous standard (GAPDH) was calculated for each sample, and analyses were performed in triplicate, yielding transcriptional quantification of gene products relative to GAPDH. 


\section{Cellular Physiology Cell Physiol Biochem 2018;49:706-716 \begin{tabular}{l|l|l} 
and Biochemistry Published online: 30 August, 2018 & $\begin{array}{l}\text { C } 2018 \text { The Author(s). Published by S. Karger AG, Basel } \\
\text { www.karger.com/cpb }\end{array}$ \\
\hline
\end{tabular}}

Tian et al.: Effects of Cacc Inhibitors on CF Function

The primers for the target genes were designed with Oligo 6.0 software (Molecular Biology Insights, Inc., Cascade, CO, USA), and synthesis was performed by Sangon Biotech Co., Ltd. (Shanghai, China). Primer sequences and expected amplicon sizes of the target genes are provided in Table 1.

Protein extraction and western blot analysis

Different groups of CFs were harvested into a lysis buffer $(20 \mathrm{mM}$ Tris-base, $137 \mathrm{mM} \mathrm{NaCl}, 10 \%$ glycerol, 1\% Triton X-100, 2 mM EDTA, and $1 \mu \mathrm{L} / \mathrm{mL}$ protease inhibitor). The lysates were centrifuged at $4{ }^{\circ} \mathrm{C}$ and $13000 \times g$ for $30 \mathrm{~min}$. The supernatant was collected, and protein concentrations were measured with a bicinchoninic acid protein assay kit (Beyotime). The proteins were separated by $8 \%$ sodium dodecyl sulfatepolyacrylamide gel electrophoresis and transferred to a nitrocellulose membrane. After blocking in trisbuffered saline (TBS) containing 5\% non-fat milk, the membranes was incubated with appropriate primary antibodies consisting of rabbit polyclonal anti-ANO1 (1:1; Abcam; Cambridge, UK) or mouse monoclonal anti- $\alpha$-smooth muscle actin ( $\alpha$-SMA; Sigma-Aldrich) overnight at $4{ }^{\circ} \mathrm{C}$. After incubation, the membrane was rinsed 3 times with TBS Tween 20 (TBST; $150 \mathrm{mM} \mathrm{NaCl,} 20 \mathrm{mM}$ Tris-base, and 0.05\% Tween 20) for 10 min, and incubated with secondary antibodies at room temperature for $1-2 \mathrm{~h}$. The membrane was washed twice with TBST for $10 \mathrm{~min}$ per wash and once with TBS for $10 \mathrm{~min}$. Protein bands were visualized on an ECL system (Amersham Biosciences, Piscataway, NJ, USA) and the results were analyzed with Image-Pro Plus 6.0 software.

\section{Immunofluorescence staining}

CFs were placed in 24-well plates at a density of $2.0 \times 10^{5}$ cells $/ \mathrm{mL}$ and $500 \mu \mathrm{L} /$ well. The cells were fixed with $10 \%$ formalin for 20 min when they reached $80 \%$ confluence. After washing 3 times with phosphate-buffered saline (PBS), The cells were permeabilized in $0.1 \%$ Triton X-100 for 20 min and blocked with $5 \%$ bovine serum albumin in TBS for $1 \mathrm{~h}$. The cells were incubated with appropriate primary antibodies at $4{ }^{\circ} \mathrm{C}$ overnight, and then with appropriate second antibodies (FITC-conjugated affiniPure goat anti-mouse $\lg \mathrm{G}[\mathrm{H}+\mathrm{L}], 1: 500$; $\mathrm{Cy}^{\mathrm{TM}} 3$-conjugated affiniPure goat anti-rabbit $\operatorname{lgG}[\mathrm{H}+\mathrm{L}], 1: 500$; Jackson ImmunoResearch Laboratories, Inc., West Grove, PA, USA) for $2 \mathrm{~h}$ at room temperature. The nuclei were stained with 4',6-diamidino-2-phenylindole (Sigma-Aldrich) for $10 \mathrm{~min}$. Protein localization in the cells was observed and captured with a laser scanning confocal microscope (LSM 510; Zeiss).

\section{Measurement of collagen secretion in rat CFs by hydroxyproline assay}

The effects of the inhibitors on collagen secretion by CFs were assessed by using a hydroxyproline assay kit (Jiancheng Bioengineering Institute, Nanjing, China) according to the manufacturer's instructions. Collagen content in the culture medium was calculated based on the absorbance, which was normalized using a blank control. Average hydroxyproline content was normalized by OD value representing cell number.

\section{Cell cycle analysis}

Cell cycle distribution was detected with a propidium iodide cell cycle kit (MultiSciences Biotech, Hangzhou, China) according to the manufacturer's instructions. Briefly, the cells were detached using $0.125 \%(\mathrm{w} / \mathrm{v}$ ) trypsin, collected by centrifugation for $10 \mathrm{~min}$ at $1000 \times \mathrm{g}$, washed with cold PBS, and fixed overnight in $70 \%$ ice-cold ethanol. The fixed cells were washed twice with PBS and treated with $10 \mu \mathrm{g} /$ $\mathrm{mL}$ RNase I for $30 \mathrm{~min}$ at $37{ }^{\circ} \mathrm{C}$ before staining with $50 \mu \mathrm{g} / \mathrm{mL}$ propidium iodide. The stained cells were analyzed by flow cytometry (Becton Dickinson, Franklin Lakes, NJ, USA).
Table 1. Oligonucleotide primers for qRT-PCR in this study. GAPDH, glyceraldehyde-3-phosphate dehydrogenase

\begin{tabular}{lccc}
\hline Primer & Gene Accession No. & Oligonucleotide Sequences (5'-3') & Amplicon Size (bp) \\
\hline ANO1F & NM_001107564 & ATTTCACCAATCTTGTCTCCATCA & 424 \\
ANO1R & & TGATAACTCCAAGAACGATTGCA & \\
collagen IF & NM_053304.1 & TGGCCCCATTGGTAACGTTG & 130 \\
collagen IR & & TCCAGCATTTCCAGAGGGACC & \\
collagen IIF & NM_032085.1 & GAGCGGAGAATACTGGGTTGA & 289 \\
collagen IIR & & TGTAATGTTCTGGGAGGCCC & \\
$\alpha$-SMAF & NM_031004.2 & CATCACCAACTGGGACGACA & \multirow{2}{*}{96} \\
$\alpha$-SMAR & & TCCGTTAGCAAGGTCGGATG & \\
GAPDHF & NM_017008.4 & ACACCCACTCCTCCACCTTT & 166 \\
GAPDHR & & TTACTCCTTGGAGGCCATGT & \\
\hline
\end{tabular}




\section{Cellular Physiology Cell Physiol Biochem 2018:49:706-716 \begin{tabular}{ll|l} 
and Biochemistry & $\begin{array}{l}\text { DOI: 10.1159/000493036 } \\
\text { Published online: } 30 \text { August, } 2018\end{array}$ & $\begin{array}{l}\text { (c) } 2018 \text { The Author(s). Published by S. Karger AG, Basel } \\
\text { www.karger.com/cpb }\end{array}$
\end{tabular}

Cell proliferation assay

The cells were seeded in 96-well plates at a density of $1.0 \times 10^{5}$ cells/well in $100 \mu \mathrm{L}$ culture medium and maintained in a $\mathrm{CO}_{2}$ incubator at $37{ }^{\circ} \mathrm{C}$ for $24 \mathrm{~h}$. Cell proliferation was measured with a Cell Counting Kit-8 (CCK-8; Beyotime). Briefly, $10 \mu \mathrm{L}$ of the CCK-8 solution was added to each well of the plate, and cells were incubated for $2 \mathrm{~h}$ at $37{ }^{\circ} \mathrm{C}$. Absorbance was measured at $450 \mathrm{~nm}$ with a microplate reader (Thermo Fisher Scientific). In this experiment, cell viability was determined at $48 \mathrm{~h}$ after treatment with $10 \mu \mathrm{M}$ T16Ainh-A01, $30 \mu \mathrm{M}$ CaCCinh-A01, or 0.1\% DMSO (control).

\section{Cell migration assay}

Transwell inserts with 8.0- $\mu$ m pore size (Corning, Inc., Corning, NY, USA) were used to assess CF migration. Prior to seeding into the upper compartments of the inserts $\left(2.0 \times 10^{5}\right.$ cells/well), CFs were prestarved in serum-free medium for $24 \mathrm{~h}$. The cells were cultured in the upper compartments for $24 \mathrm{~h}$, the non-invasive cells were removed from the upper surface of each insert, and the migrated cells were fixed with methanol, stained with $1 \%$ crystal violet, and counted using a light microscope in 5 random visual fields at a magnification of $100 \times$.

\section{Statistical analysis}

All data are expressed as the mean \pm standard error of the mean. Statistical analyses were performed using GraphPad Prism software (GraphPad Software, Inc., San Diego, CA, USA). An unpaired Student's $t$-test or one-way analysis of variance followed by Bonferroni's multiple comparisons post hoc test was used for statistical comparisons. Differences were considered statistically significant at $p<0.05$.

\section{Results}

\section{Identification of CFs and expression of ANO1 in CFS}

CFs stained positive for vimentin and were typically spindle or triangle shaped under a light microscope (Fig. 1A). Next, we investigated the distribution of ANO1 protein in CFs by immunostaining. As shown in Fig. 1B, ANO1 expression was predominantly located on the nuclear membrane and partially around the nucleus, especially in CFs in the mitotic phase. To our knowledge, this is the first report on the cellular localization of ANO1 protein in CFs.

\section{T16Ainh-A01 and CaCCinh-A01 reduce $\left[\mathrm{Cl}^{-}\right]_{i}$ in $\mathrm{CFs}$ but do not affect $\left[\mathrm{Ca}^{2+}\right]_{i}$}

As shown in Fig. 2A (upper) and Fig. 2B, there was no significant difference of $\left[\mathrm{Ca}^{2+}\right]$ i among the cells treated with $10 \mu \mathrm{M}$ T16Ainh-A01, $30 \mu \mathrm{M}$ CaCCinh-A01 and 0.1\% DMSO, respectively $(p>0.05)$. This indicates that neither compounds had an effect on $\left[\mathrm{Ca}^{2+}\right]_{\mathrm{i}}$. The novel fluorescent indicator MQAE was used to measure $\left[\mathrm{Cl}^{-}\right]_{i}$ in $\mathrm{CFs}$. High fluorescence intensity of MQAE reflects low $\left[\mathrm{Cl}^{-}\right]_{\text {i, }}$, while low fluorescence intensity reflects high $\left[\mathrm{Cl}^{-}\right]$ i. As shown in Fig. 2A (lower panel), T16Ainh-A01 inhibited $\mathrm{Cl}^{-}$influx into the nucleus, while CaCCinh-A01 led to a decrease of $\mathrm{Cl}^{-}$content in intracellular compartments around the nucleus $\left[\mathrm{Cl}^{-}\right]_{\mathrm{i}}$ was significantly decreased in CFs treated with both T16Ainh-A01 and CaCCinh-A01 (both $p<0.01, n=5$, Fig. 2C).

Fig. 1. Identification of the phenotype of rat cardiac fibroblasts (CFs) and the expression of ANO1. (A) vimentin immunostaining (red). Scale bar = $50 \mu \mathrm{m}$. (B) ANO1 immunostaining (red). ANO1 localized predominantly at the nuclear membrane and partially within intracellular compartments around nucleus in $\mathrm{CF}$, especially at mitotic phase (indicated by white arrows). Scale bar $=25 \mu \mathrm{m}$.

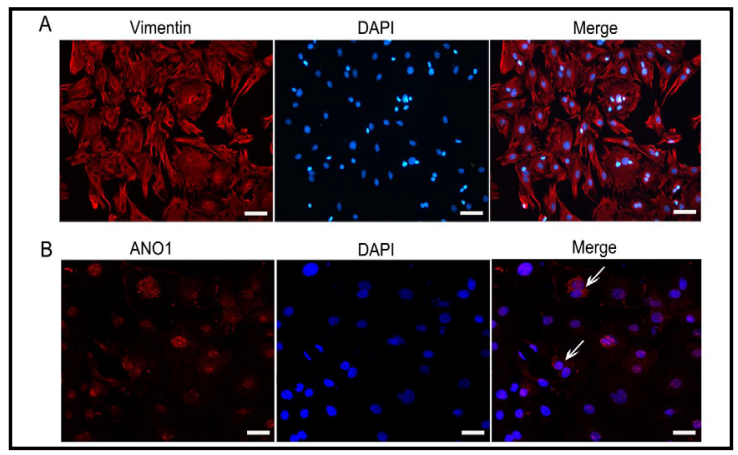




\section{Cellular Physiology \begin{tabular}{ll|l} 
DOI: 10.1159/000493036 & $\begin{array}{l}\text { O } 2018 \text { The Author(s). Published by S. Karger AG, Basel } \\
\text { wwwwkarger.com/cpb }\end{array}$
\end{tabular} \\ Tian et al.: Effects of Cacc Inhibitors on CF Function}

Fig. 2. The effects of T16Ainh-A01 and CaCCinh-A01 on intracellular $\mathrm{Ca}^{2+}$ and $\mathrm{Cl}^{-}$concentrations in primary $\mathrm{CFs}$. (A) intracellular $\mathrm{Ca}^{2+}$ and $\mathrm{Cl}^{-}$were detected by Fluo 4-AM (upper) and MQAE probe staining (lower), respectively. (B-C) quantitative analysis of intracellular $\mathrm{Ca}^{2+}$ and $\mathrm{Cl}^{-}$ density. Data are expressed as mean $\pm \mathrm{SEM}$; $n=5$; ns, no significant difference, ${ }^{* *} \mathrm{P}<0.01$ represents statistical comparisons of inhibitors versus controls. Scale bar $=$ $25 \mu \mathrm{m}$.
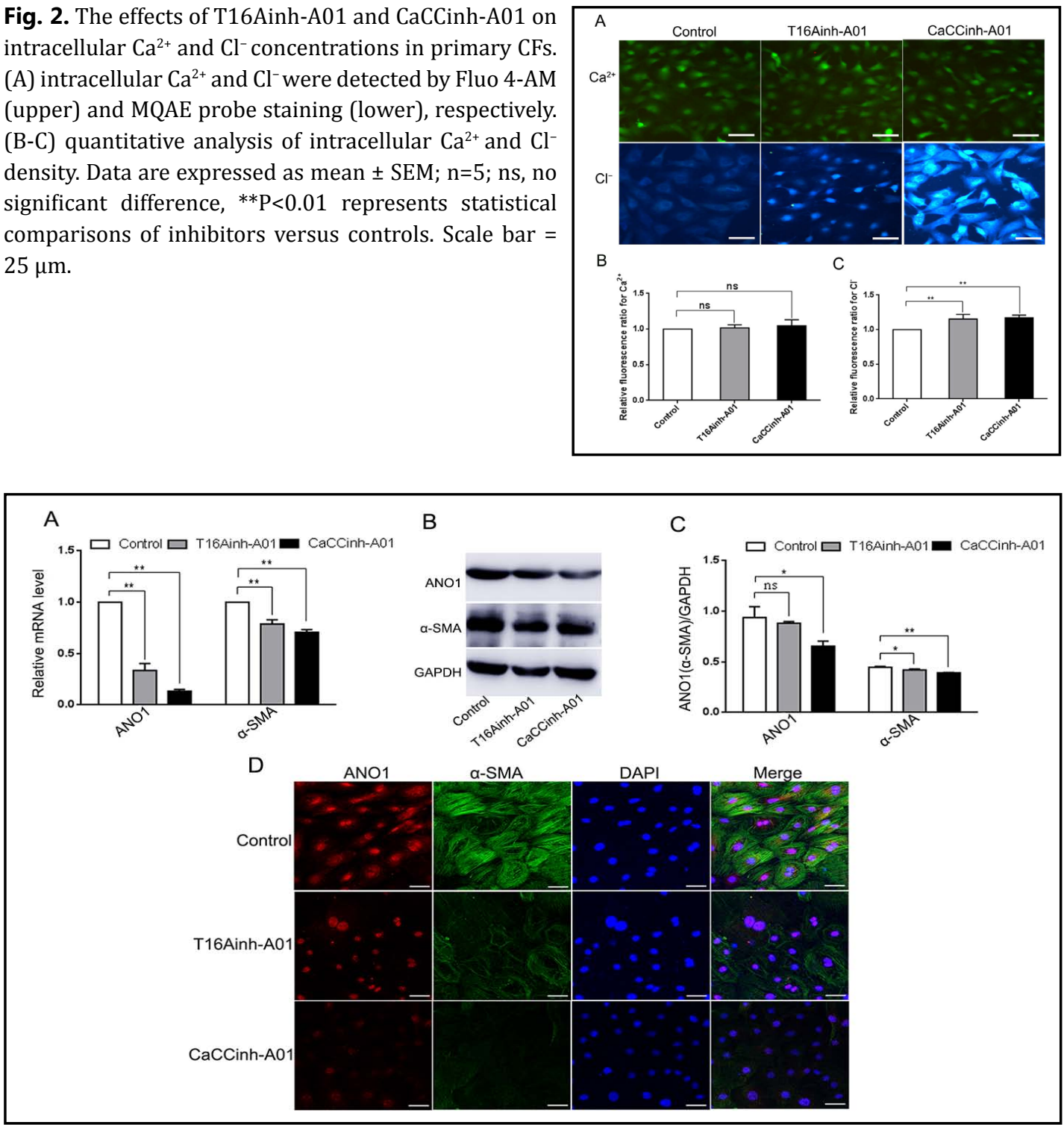

Fig. 3. The expression of ANO1 and $\alpha$-SMA in rat CFs after treatment with T16Ainh-A01 and CaCCinh-A01 for $48 \mathrm{~h}$. (A) qRT-PCR data show the mRNA expressions of AN01 and $\alpha$-SMA. (B-C) Western blot data show the protein expressions of ANO1 and $\alpha$-SMA. (D) expression of AN01 and $\alpha$-SMA by immunoflurescence staining. ANO1 and $\alpha$-SMA were red and green, respectively, and nuclei were identified by DAPI staining (blue). ns, no significant difference; ${ }^{*} \mathrm{p}<0.05$, vs. control; ${ }^{* *} \mathrm{p}<0.01$, vs. control. Scale bar $=40 \mu \mathrm{m}$.

\section{Effect of T16Ainh-A01 and CaCCinh-A01 on ANO1 and $\alpha$-SMA expression in rat CFs}

When the primary CFs were treated with $10 \mu \mathrm{M}$ T16Ainh-A01, the expression of AN01 and $\alpha$-SMA mRNA was markedly decreased compared with control $(p<0.01$ for both ANO1 and $\alpha$-SMA, $n=5$, Fig. 3A). The inhibitory effects of $30 \mu$ M CaCCinh-A01 on AN01 and $\alpha$-SMA mRNA expression were even greater $(p<0.01, n=5$, Fig. 3A). Western blot analysis showed that the protein expressions of ANO1 and $\alpha$-SMA protein in CFs was markedly reduced in CFs after treatment with CaCCinh-A01 ( $p<0.05$ for AN01, $p<0.01$ for $\alpha$-SMA, $n=5$ ). T16Ainh-A01 also reduced the protein expression of $\alpha$-SMA, but did not affect AN01 protein expression ( $p>0.05, n=5$, Fig. 3B and C). Consistent with the western blot data, immunofluorescence staining also showed that CaCCinh-A01 caused comparatively weaker fluorescent signals for ANO1 and $\alpha$-SMA in CFs compared with T16Ainh-A01 (Fig. 3D). 


\section{Cellular Physiology Cell Physiol Biochem 2018;49:706-716

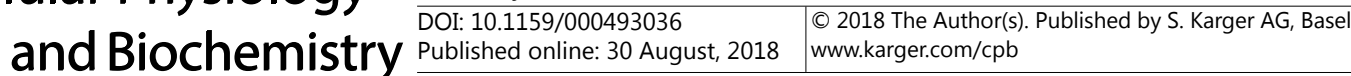

Fig. 4. Collagen secretion from CFs after treatment with T16Ainh-A01 and CaCCinh-A01 for 48 h. (A) collagen content (measured by hydroxyproline assay) in different groups. (B) relative mRNA expressions of collagen I and collagen III (measured by qRT-PCR) in different groups. Data are expressed as mean \pm SEM, ${ }^{* *} \mathrm{p}<0.01$, vs. control.

Fig. 5. The effects of T16Ainh-A01 and CaCCinh-A01 on proliferation and cell cycle of rat CFs. (A-D) flow cytometric data show cell cycle phases in different groups of CFs. (E) cell proliferation (measured by CCK-8 assay). CFs in T16Ainh-A01 and CaCCinh-A01 groups were treated with T16Ainh-A01 (10 $\mu \mathrm{M})$ and CaCCinh-A01 (30 $\mu \mathrm{M})$ for $48 \mathrm{~h}$, respectively. Treatments with T16Ainh-A01 and CaCCinh-A01 markedly arrested the cells at G1 phase and caused the reduction of cells at $\mathrm{S}$ phase. Data are expressed as mean \pm SEM; ns, no significant difference; ${ }^{* *} \mathrm{p}<0.01$, vs. control.
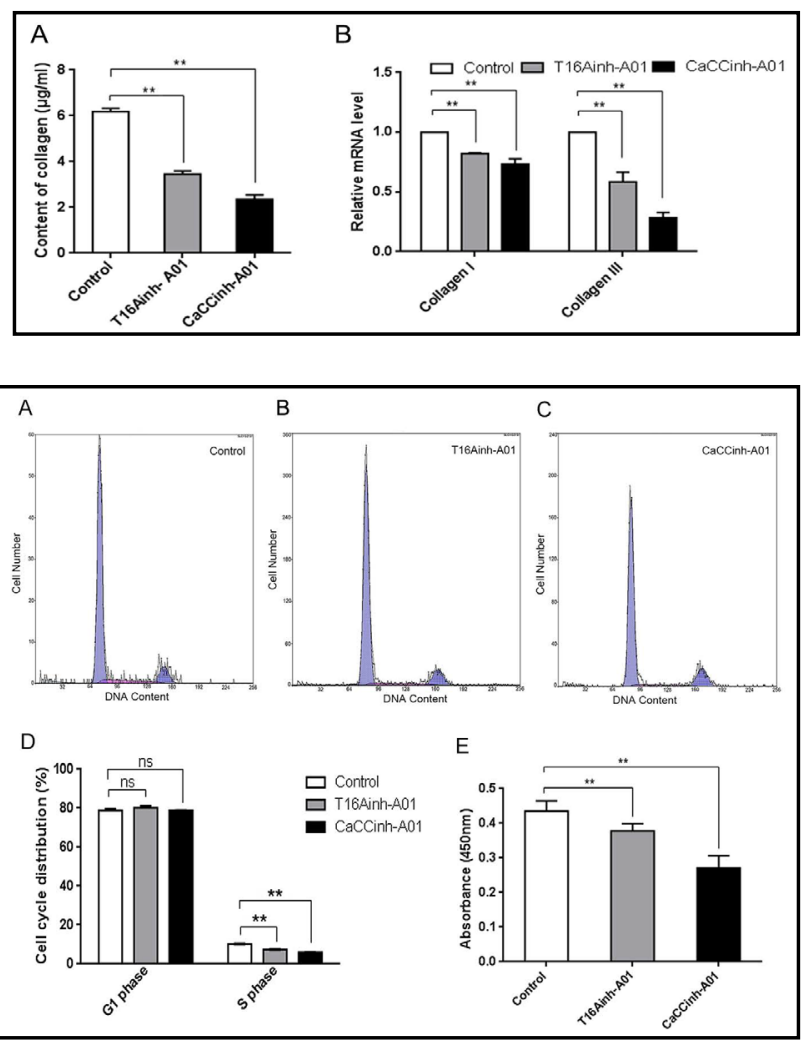

T16Ainh-A01 and CaCCinh-A01 reduced the secretion of collagen from CFs

The amounts of secreted collagen $(\mu \mathrm{g} / \mathrm{mL})$ secreted were significantly lower in the T16Ainh-A01 and CaCCinh-A01-treated groups compared with the control group (T16Ainh-A01 $3.453 \pm 0.137$ vs. $6.180 \pm 0.146$ of control, $p<0.01, n=5$; CaCCinh-A01 $2.355 \pm 0.187$ vs. $6.180 \pm 0.146$ of control, $p<0.01, n=5$, Fig. 4 A). Both CaCCinh-A01 and T16Ainh-A01 displayed significant inhibitory effects on the mRNA expression of types I and type III collagen (both $p<0.01, n=5$, Fig. 4B).

T16Ainh-A01 and CaCCinh-A01 inhibited CF proliferation and cell cycle progression

Both compounds inhibited cell cycle progression at the G1 phase and there was a significant decrease in the number of cells at the $S$ phase. The percentages of cells at the G1 phase were $80.18 \pm 0.49 \%$ and $78.87 \pm 0.07 \%$ (vs. $78.99 \pm 0.31 \%$ in control, $n=3$ ) in the T16Ainh-A01- and CaCCinh-A01-treated groups, respectively. The percentages of the cells at the $S$ phase decreased significantly from $10.18 \pm 0.06 \%$ in the control group to $7.41 \pm 0.02 \%$ $(n=3)$ and $6.04 \% \pm 0.04 \%(n=3)$ in the T16Ainh-A01 and CaCCinh-A01-treated groups (both $p<0.01, n=3$, Fig. 5A-D), respectively. Both T16Ainh-A01 and CaCCinh-A01 exhibited significant inhibitory effects on CF proliferation (T16Ainh-A01 absorbance $0.378 \pm 0.0097$ vs. $0.435 \pm 0.0133$ of control, $p<0.01, n=3$; CaCCinh-A01 absorbance $0.271 \pm 0.0155$ vs. $0.435 \pm 0.0133$ of control, $n=3$, Fig. 5E).

\section{T16Ainh-A01 and CaCCinh-A01 inhibited CF migration}

The migration of CFs was measured by a Transwell migration assay. As shown in Fig. 6A and $\mathrm{B}$, both T16Ainh-A01 and CaCCinh-A01 significantly inhibited the migration of rat CFs (both $p<0.01, n=5$ ). 


\section{Cellular Physiology Cell Physiol Biochem 2018;49:706-716

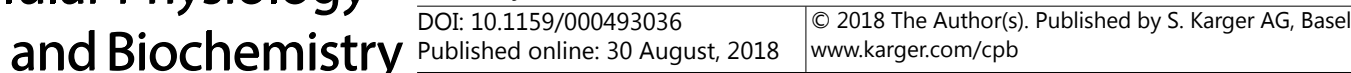 \\ Tian et al.: Effects of Cacc Inhibitors on CF Function}

\section{Discussion}

We showed for the first time that ANO1 was mainly localized in the nuclear membrane of the rat CFs. T16Ainh-A01 inhibited $\mathrm{Cl}^{-}$influx into the nucleus, and CaCCinh-A01 caused a global decrease of $\mathrm{Cl}^{-}$ content. Our findings demonstrated that both inhibitors suppressed CF proliferation by inducing cell cycle arrest at the G1 phase, and also inhibited cell migration and collagen secretion of CFs.

ANO1 is a key factor for CaCCmediated conductance [13-15]. AN01 localization may vary in distinct cell types [22-24]. In dorsal root ganglion neurons. ANO1 is predominately located in the cell membrane [25]. A recent study on the expression, localization, and distribution of ANO1 in murine ventricular tissues and myocytes revealed that ANO1 was evenly distributed in the left ventricular epicardium and endocardium and localized abundantly to the plasma membrane of murine ventricular myocytes [24]. However, in cultured uterine smooth muscle cells, ANO1 was found to be expressed mainly in the cytoplasm adjacent to the nucleus [26]. In this study, we demonstrated for the first time that ANO1 was predominantly expressed on the nuclear membrane of CFs and partially within intracellular compartments around the nucleus. This result challenges a previous study demonstrating that ANO1 was highly expressed in the cytoplasmic membrane of rat CFs [11]. Notably, ANO1 expression was extremely low in freshly isolated CFs, but it was significantly enhanced at $48 \mathrm{~h}$ after $\mathrm{CF}$ attachment to the culture dish (data not shown). The expression pattern of ANO1 indicates that it not only plays an important role in the modulation of $\mathrm{Cl}^{-}$ flux through CaCCs but also has additional biological functions.

Several studies have confirmed that T16Ainh-A01 and CaCCinh-A01 exert their inhibitory effects in a dose-dependent manner [18, 19, 27, 28]. T16Ainh-A01 $(10 \mu \mathrm{M})$ strongly inhibits TMEM16A-mediated iodide influx and completely blocks CaCC conductance in salivary gland cells. CaCCinh-A01, with fitted $\mathrm{IC}_{50}$ values of $2.1 \mu \mathrm{M}$, can block ANO1-encoded CaCC currents at a high concentration $(30 \mu \mathrm{M})$ but has no effect on $\left[\mathrm{Ca}^{2+}\right]_{\mathrm{i}}$ in human intestinal epithelial cells $[18,29]$. In the present study, we also found that T16Ainh-A01 and CaCCinh-A01 exerted their inhibitory effects in a dose-dependent manner. Exposure to $20 \mu \mathrm{M} \mathrm{T} 16 \mathrm{Ainh}-\mathrm{A} 01$ and 60 $\mu \mathrm{M}$ CaCCinh-A01 was cytotoxic, as manifested by cells death and morphology changes (data not shown), whereas $10 \mu \mathrm{M}$ T16Ainh-A01 and $30 \mu \mathrm{M}$ CaCCinh-A01 did not show obvious cytotoxicity and strongly inhibited $\left[\mathrm{Cl}^{-}\right]_{\mathrm{i}}$ and the proliferation of CFs.

It has been reported that T16Ainh-A01 can inhibit ANO1-dependent chloride conductance in cells but does not affect ANO1 expression in airway and intestinal epithelia [18], whereas CaCCinh-A01 inhibits ANO1-dependent cell proliferation by promoting the endoplasmic reticulum-associated proteasomal degradation of ANO1 in ANO1-dependent cell lines [17]. It has also been reported that ANO1 plays an important role in the modulation of cardiac fibrosis and is a potential therapeutic target for cardiac fibrosis of myocardial infarction [11]. However, little is known about the effects of T16Ainh-A01 and CaCCinh-A01 on ANO1 expression in CFs. In the present study, we found that CaCCinh-A01 significantly inhibited ANO1 expression, whereas T16Ainh-A01 did not have a significantly effect on the protein level of ANO1, although both inhibitors could suppress CF proliferation, migration, 
and collagen secretion. We speculate that T16Ainh-A01 may affect CF functions by simply inhibiting CaCCs, whereas CaCCinh-A01 exerts its inhibitory effects on CFs by not only closing CaCCs but also by inactivating AN01. The precise mechanisms responsible for the actions of these inhibitors require further investigation.

Further, we evaluated the effects of T16Ainh-A01 and CaCCinh-A01 on the expression of $\alpha$-SMA, a typical marker of myofibroblasts, which are closely associated with cardiac fibrosis [30, 31], and demonstrated that both inhibitors reduced $\alpha$-SMA expression at the mRNA and protein level. The synthesis and secretion of collagen are the main functions of $\mathrm{CFs}$, which play a pivotal role in the myocardial repair process [32]. Our results showed that CaCCinh-A01 and T16Ainh-A01 could significantly inhibit the secretion of collagen from CFs. These findings indicate that both inhibitors may play roles in modulating the development of cardiac fibrosis.

Chloride channels provide the main electrical shunt for the acidification of most organelles of the secretory and endocytic pathways and play essential roles in a variety of physiological and pathophysiological processes in many diseases [33]. CaCCinh-A01 and T16Ainh-A01 are the most potent inhibitors of calcium-activated $\mathrm{Cl}^{-}$conductance and are typically used to examine the contributions of CaCCs/ANO1 in physiological models, although their specificity remains uncertain $[8,16]$. CaCCinh-A01 can completely block CaCC current in human bronchial and intestinal epithelial cells, whereas T16Ainh-A01 poorly inhibits total CaCC current, but blocks an initial agonist-stimulated transient chloride current [34]. CaCCs are activated by an increases in the level of intracellular free calcium, and $\mathrm{Cl}^{-}$flux through CaCCs is generally thought to be driven by ANO1 or ANO2 [8]. An important finding of this study was that T16Ainh-A01 inhibited $\mathrm{Cl}^{-}$influx into the nucleus, while CaCCinh-A01 reduced $\mathrm{Cl}^{-}$level in intracellular compartments around the nucleus. These results extend our knowledge of the mechanisms of action of two new inhibitors and lay the foundation for the future development of anti-fibrotic drugs.

\section{Conclusion}

Overall, this study provides novel information about the effects of T16Ainh-A01 and CaCCinh-A01 on CFs. Both inhibitors can suppress cell proliferation, cell migration, and collagen secretion from CFs, suggesting that they have therapeutic potential for the treatment of cardiac fibrosis. However, their involvement and utility in heart disease remain unclear and require further study.

\section{Acknowledgements}

Authors would like to thank Dr Magomed Khaidakov from University of Arkansas for Medical Sciences, for revising the paper and advice in the work. The authors gratefully acknowledge funding support by the National Natural Science Foundation of China (Grant No. 81560081).

\section{Disclosure Statement}

The authors declare to have no conflict of interests.

\section{References}

1 Travers JG, Kamal FA, Robbins J, Yutzey KE, Blaxall BC: Cardiac fibrosis: the fibroblast awakens. Circ Res 2016;118:1021-1040. 


\section{Cellular Physiology Cell Physiol Biochem 2018:49:706-716 \begin{tabular}{l|l|l|l} 
and Bioch 10.1159/000493036 & 2018 The Author(s). Published by S. Karger AG, Basel
\end{tabular} and BiOChemistry Published online: 30 August, 2018 www.karger.com/cpb}

Tian et al.: Effects of Cacc Inhibitors on CF Function

-2 Shi H, Zhang X, He Z, Wu Z, Rao L, Li Y: Metabolites of hypoxic cardiomyocytes induce the migration of cardiac fibroblasts. Cell Physiol Biochem 2017;41:413-421.

-3 Chistiakov DA, Orekhov AN, Bobryshev YV: The role of cardiac fibroblasts in post-myocardial heart tissue repair. Exp Mol Pathol 2016;101:231-240.

-4 Deng P, Chen L, Liu Z, Ye P, Wang S, Wu J, Yao Y, Sun Y, Huang X, Ren L, Zhang A, Wang K, Wu C, Yue Z, Xu X, Chen M: MicroRNA-150 inhibits the activation of cardiac fibroblasts by regulating c-Myb. Cell Physiol Biochem 2016;38:2103-2122.

5 Chacar S, Fares N, Bois P, Faivre JF: Basic signaling in cardiac fibroblasts. J Cell Physiol 2017;232:725-730.

-6 Liu W, Lu M, Liu B, Huang Y, Wang K: Inhibition of $\mathrm{Ca}^{2+}$-activated $\mathrm{Cl}^{-}$channel AN01/TMEM16A expression suppresses tumor growth and invasiveness in human prostate carcinoma. Cancer Lett 2012;326:41-51.

7 Nguyen MN, Kiriazis H, Gao XM, Du XJ: Cardiac fibrosis and arrhythmogenesis. Compr Physiol 2017;7:10091049.

-8 Friard J, Tauc M, Cougnon M, Compan V, Duranton C, Rubera I: Comparative Effects of Chloride Channel Inhibitors on LRRC8/VRAC-Mediated Chloride Conductance. Front Pharmacol 2017;8:328.

-9 El Chemaly A, Norez C, Magaud C, Bescond J, Chatelier A, Fares N, Findlay I, Jayle C, Becq F, Faivre JF, Bois P: ANO1 contributes to angiotensin-II-activated $\mathrm{Ca}^{2+}$-dependent $\mathrm{Cl}^{-}$current in human atrial fibroblasts. J Mol Cell Cardiol 2014;68:12-19.

10 He ML, Liu WJ, Sun HY, Wu W, Liu J, Tse HF, Lau CP, Li GR: Effects of ion channels on proliferation in cultured human cardiac fibroblasts. J Mol Cell Cardiol 2011;51:198-206.

11 Gao Y, Zhang YM, Qian LJ, Chu M, Hong J, Xu D: AN01 inhibits cardiac fibrosis after myocardial infraction via TGF-beta/smad3 pathway. Sci Rep 2017;7:2355.

12 Gourdie RG, Dimmeler S, Kohl P: Novel therapeutic strategies targeting fibroblasts and fibrosis in heart disease. Nat Rev Drug Discov 2016;15:620-638.

-13 Yang YD, Cho H, Koo JY, Tak MH, Cho Y, Shim WS, Park SP, Lee J, Lee B, Kim BM, Raouf R, Shin YK, Oh U: TMEM16A confers receptor-activated calcium-dependent chloride conductance. Nature 2008;455:12101215.

14 Schroeder BC, Cheng T, Jan YN, Jan LY: Expression cloning of TMEM16A as a calcium-activated chloride channel subunit. Cell 2008;134:1019-1029.

15 Caputo A, Caci E, Ferrera L, Pedemonte N, Barsanti C, Sondo E, Pfeffer U, Ravazzolo R, Zegarra-Moran O, Galietta LJ: TMEM16A, a membrane protein associated with calcium-dependent chloride channel activity. Science 2008;322:590-594.

16 Boedtkjer DM, Kim S, Jensen AB, Matchkov VM, Andersson KE: New selective inhibitors of calcium-activated chloride channels-T16A(inh)-A01, CaCC(inh)-A01 and MONNA-what do they inhibit? Br J Pharmacol 2015;172:4158-4172.

17 Bill A, Hall ML, Borawski J, Hodgson C, Jenkins J, Piechon P, Popa O, Rothwell C, Tranter P, Tria S, Wagner T, Whitehead L, Gaither LA: Small molecule-facilitated degradation of AN01 protein: a new targeting approach for anticancer therapeutics. J Biol Chem 2014;289:11029-11041.

18 Namkung W, Phuan PW, Verkman AS: TMEM16A inhibitors reveal TMEM16A as a minor component of calcium-activated chloride channel conductance in airway and intestinal epithelial cells. J Biol Chem 2011;286:2365-2374.

19 Guan L, Song Y, Gao J, Gao J, Wang K: Inhibition of calcium-activated chloride channel AN01 suppresses proliferation and induces apoptosis of epithelium originated cancer cells. Oncotarget 2016;7:78619-78630.

20 Rhaleb N, Peng H, Harding P, Tayeh M, LaPointe M, Carretero O: Effect of N-acetyl-seryl-aspartyl-lysyl-proline on DNA and collagen synthesis in rat cardiac fibroblasts. Hypertension 2001;37:827-832.

21 Chang Y, Guo K, Li Q Li C, Guo Z, Li H: Multiple directional differentiation difference of neonatal rat fibroblasts from six organs. Cell Physiol Biochem 2016;39:157-171.

-22 Tian Y, Schreiber R, Kunzelmann K: Anoctamins are a family of $\mathrm{Ca}^{2+}$-activated $\mathrm{Cl}^{-}$channels. J Cell Sci 2012;125:4991-4998.

23 Rock J, Futtner C, Harfe B: The transmembrane protein TMEM16A is required for normal development of the murine trachea. Dev Biol 2008;321:141-149.

24 Ye Z, Wu MM, Wang CY, Li YC, Yu CJ, Gong YF, Zhang J, Wang QS, Song BL, Yu K, Hartzell HC, Duan DD, Zhao D, Zhang ZR: Characterization of cardiac anoctamin $1 \mathrm{Ca}^{2+}$-activated chloride channels and functional role in ischemia-induced arrhythmias. J Cell Physiol 2015;230:337-346. 


\section{Cellular Physiology Cell Physiol Biochem 2018;49:706-716 \begin{tabular}{l|l} 
DOI: 101159/000493036 & $\begin{array}{l}\text { O 2018 The Author(s). Published by S. Karger AG, Basel } \\
\text { www.karger.com/cpb }\end{array}$
\end{tabular} \\ Tian et al.: Effects of Cacc Inhibitors on CF Function}

25 Zhao L, Li LI, Ma KT, Wang Y, Li J, Shi WY, Zhu HE, Zhang ZS, Si JQ: NSAIDs modulate GABA-activated currents via $\mathrm{Ca}(2+)$-activated $\mathrm{Cl}(-)$ channels in rat dorsal root ganglion neurons. Exp Ther Med 2016;11:1755-1761.

-26 Bernstein K, Vink JY, Fu XW, Wakita H, Danielsson J, Wapner R, Gallos G: Calcium-activated chloride channels anoctamin 1 and 2 promote murine uterine smooth muscle contractility. Am J Obstet Gynecol 2014;211:688 e681-610.

27 Zhang X, Li H, Zhang H, Liu Y, Huo L, Jia Z, Xue Y, Sun X, Zhang W: Inhibition of transmembrane member 16A calcium-activated chloride channels by natural flavonoids contributes to flavonoid anticancer effects. $\mathrm{Br} \mathrm{J}$ Pharmacol 2017;174:2334-2345.

-28 Mazzone A, Eisenman ST, Strege PR, Yao Z, Ordog T, Gibbons SJ, Farrugia G: Inhibition of cell proliferation by a selective inhibitor of the $\mathrm{Ca}^{2+}$-activated $\mathrm{Cl}^{-}$channel, Ano1. Biochem Biophys Res Commun 2012;427:248253.

29 De La Fuente R, Namkung W, Mills A, Verkman AS: Small-molecule screen identifies inhibitors of a human intestinal calcium-activated chloride channel. Mol Pharmacol 2008;73:758-768.

30 Hong Y, Cao H, Wang Q, Ye J, Sui L, Feng J, Cai X, Song H, Zhang X, Chen X: MiR-22 may suppress fibrogenesis by targeting TGFbetaR I in cardiac fibroblasts. Cell Physiol Biochem 2016;40:1345-1353.

-31 Guo Y, Dong Z, Shi Y, Wang W, Wang L, Sun J, Sun X, Tian Z, Yao J, Li Z, Cheng J, Tian Y: Sonodynamic therapy inhibits fibrogenesis in rat cardiac fibroblasts induced by TGF-beta1. Cell Physiol Biochem 2016;40:579588 .

-32 Shinde AV, Frangogiannis NG: Fibroblasts in myocardial infarction: a role in inflammation and repair. J Mol Cell Cardiol 2014;70:74-82.

-33 Stauber T, Jentsch TJ: Chloride in vesicular trafficking and function. Annu Rev Physiol 2013;75:453-477.

-34 Hwang SJ, Basma N, Sanders KM, Ward SM: Effects of new-generation inhibitors of the calcium-activated chloride channel anoctamin1 on slow waves in the gastrointestinal tract. Br J Pharmacol 2016;173:13391349. 